\title{
A Novel Immune Checkpoint-Related Gene Signature to Predict Overall Survival and Immunotherapy Response in Triple-Negative Breast Cancer
}

Jingyi Liu

Xijing Hospital Department of Radiation Oncolgy

Siyuan Tian

State Key Laboratory of Cancer Biology, Xijing Hospital of Digestive Disease

Yuwei Ling

Department of General Surgery, Center for Thyroid and Breast Surgery, Xuanwu Hospital

Xinyi Zhang

Department of Nephrology, The First Affiliated Hospital of Harbin Medical University.

Yan Li

Xijing Hospital Department of Radiation Oncology

Ning Su

Xijing Hospital Department of Radiation Oncology

Mei Shi ( $\nabla$ mshi82@hotmail.com )

Xijing Hospital Department of Radiation Oncology

\section{Research Article}

Keywords: TNBC, immune checkpoint, gene signature, prognosis, immunotherapy response

Posted Date: May 21st, 2021

DOl: https://doi.org/10.21203/rs.3.rs-528492/v1

License: (c) (1) This work is licensed under a Creative Commons Attribution 4.0 International License.

Read Full License 


\section{Abstract}

Triple-negative breast cancer (TNBC) is a highly aggressive subtype of breast cancer that lacks effective therapeutic targets. Immunotherapy is considered as a novel treatment strategy for TNBC. However, only some patients could benefit from the treatment. Limited studies have comprehensively explored expression patterns and prognostic value of immune checkpoint genes (ICGs) in TNBC. In this study, we downloaded relevant ICGs expression profiles and clinical TNBC data from the Cancer Genome Atlas (TCGA) and the Molecular Taxonomy of Breast Cancer International Consortium (METABRIC) database. The least absolute shrinkage and selection operator (LASSO) Cox regression analysis was employed to develop a multi-gene signature for predicting the prognostic outcome. PDCD1, PDCD1LG2 and KIR3DL2 were identified as hub genes and incorporated into the model. This gene signature could stratify patients into two prognostic subgroups, and unfavorable clinical outcomes were observed in high-risk patients. The predictive performance was assessed by the receiver operating characteristic curves. Moreover, we also analyzed differences in immune status and therapeutic response between both groups. This novel gene signature may be served as a robust prognostic marker, but also an indicator reflecting immunotherapy response.

\section{Introduction}

Breast cancer (BC) is the most common malignant tumor, posing a serious threat to the health of women worldwide[1]. As a result of high heterogeneity of $\mathrm{BC}$, different types differ in terms of morphology, molecular pathological features, clinical characteristics and response to treatment[2,3]. Triple negative breast cancer (TNBC), a highly aggressive subtype characterized by negative expression of hormone receptors and human epidermal growth factor receptor 2 , comprises about $15-20 \%$ of all BC cases and is more common in younger patients[4,5]. Due to the paucity of effective therapeutic targets, surgery combined radiotherapy and chemotherapy is the main therapeutic approach[6]. However, the resistance of the chemoradiotherapy and the aggressive nature of TNBC trigger to the high postoperative recurrence rate which even reached $25 \%-40 \%[5]$ and the median survival time was only 20 months[7, 8]. Therefore, it's urgent to explore new therapeutic approaches to improve treatment outcomes.

Recently, immunotherapy has continued to bring breakthroughs and surprising results in the treatment of refractory tumors such as melanoma[9], lymphoma[10] and lung cancer[11], and some studies have also demonstrated immunotherapy may provide new ideas for the treatment of TNBC[12, 13]. Immune checkpoints are considered as the most important therapeutic targets and immune checkpoint inhibitors (ICls) were well-studied in different malignancies[14]. Mechanistically, ICls could block immunosuppressive receptors and strengthen the capability of cytotoxicity and proliferation of tumorinfiltrating lymphocytes (TILs)[15]. In fact, TNBC shows pronounced genomic instability and tumor mutational loads so that it appears higher tumor immunogenicity compared with other subtypes[16, 17]. Thus, TNBC is readily recognized by the immune system and expected to gain benefits from immunotherapy. However, the overall remission rate for TNBC patients remained only $5 \%-23 \%[18]$, which 
indicated that not all individuals could benefit from the immunotherapy. Therefore, a robust biomarker is needed to identify potential beneficiary population.

Several published studies revealed that immune checkpoint genes (ICGs) correlated with immunotherapy response and prognosis of patients with nasopharyngeal carcinoma, hepatocellular carcinoma and other tumors $[19,20]$. However, studies for TNBC were limited to a single immune checkpoint gene and the findings were still under debate. Few studies have systematically investigated the expression pattern of ICGs in TNBC or attempted to establish an immune checkpoint genes-based prognostic gene signature to facilitate and improve clinical decision making. In our present study, taking the advantage of genomics databases, we aimed to identify the prognostic ICGs and construct a novel gene signature for TNBC based on the transcriptomic and clinical data from the TNBC cohort in the Cancer Genome Atlas (TCGA) database and the Molecular Taxonomy of Breast Cancer International Consortium (METABRIC) database. We expected that the novel gene signature could provide more references for individual prognosis and targeted therapy in clinical work.

\section{Methods}

\section{Data source}

The transcriptome data and clinical characteristics of samples were obtained from the TCGA (https://portal.gdc.cancer.gov/) and the METABRIC database (http://molonc.bccrc.ca/aparicio$\mathrm{lab} /$ research/metabric/). All patients were required to meet the following inclusion criteria: (1) TNBC samples determined by immunochemistry results of ER, PR and HER2 status; (2) Transcriptome data and clinical data were comprehensive and available; (3) The overall survival time was longer than one month. After screening, 113 TNBC patients in the TCGA database and 286 patients in the METABRIC database were included for the subsequent analysis. The clinical characteristics of two cohorts were presented in Table 1. We used samples in the TCGA database as the training cohort and the METABRIC database was employed as the validation cohort. The overall workflow of this research is presented in Supplementary Figure 1.

\section{Differentially expressed analysis of immune-checkpoint genes}

In total, 79 ICGs were retrieved from literature, and most of the ICGs were ligands, receptors or important molecules in immune checkpoint pathways (Supplementary Table 1)[21]. The expression data of above ICGs of TNBC and normal tissues from the TCGA database were extracted. We screened out differentially expressed immune-checkpoint genes (DE-ICGs) using the "limma" package under the R environment (version 4.0). $\| \log _{2}$ Fold Changel $>1$ and $p$ value $<0.05$ were the cut-off criteria. Then, the heatmap of DEICGs was generated using the "pheatmap" R package. Volcano-plot was plotted with Graphpad Prism 7.

\section{GO and KEGG enrichment analysis}


"Cluster profiler" R package was applied to conduct GO functional annotation analysis and KEGG pathway enrichment analysis, aiming at exploring biological functions and signaling pathways that the DE-ICGs mainly participated in. GO functional analysis includes three main categories: biological processes (BP), cellular components (CC), and molecular functions (MF). The results are visualized using the "enrichplot" package.

\section{Identification of prognosis-related ICGs}

To identify the prognosis-related ICGs, we firstly matched the expression data of these ICGs with the survival information of TNBC patients. The "survival" package was used to conduct univariate Cox analysis to select the prognostic genes. Statistical significance was determined by $p<0.05$. Next, LASSO regression analysis was applied to reduce collinearity between genes and prevent over-fitting of the subsequently constructed prognostic risk model. Dimensionality reduction could be achieved by compressing the regression coefficients of independent variables. Finally, the genes identified were entered into the multivariate cox regression analysis to obtain the ICGs associated with overall survival (OS) of TNBC patients.

\section{Construction and validation of an ICGs-based prognostic gene signature}

The coefficients of hub genes in the multivariate Cox regression were used to calculate the risk score for each sample. The risk score $=\beta_{1}{ }^{*} X_{1}+\beta_{2}{ }^{*} X_{2}+\ldots+\beta_{n}{ }^{*} X_{n}$, where $X$ represents the gene expression values and $\beta$ represents the regression coefficient of each hub gene included in the ICGs signature model. The median score was considered as the cut-off value to divide the TNBC patients into a high-risk group and a low-risk group. The Kaplan-Meier survival curves were generated by R package "Survival" to further explore the association between the risk scores and prognostic outcomes. We also used "timeROC" package to plot the receiver operating characteristic (ROC) curve of the model and evaluate the accuracy of this prognostic model by calculating the area under the curve (AUC). Meanwhile, in order to verify the stability of our gene signature, external validation was also performed in the METABRIC database.

\section{Correlation analysis with immune cell infiltration and immunotherapy response}

To further elucidate the association between immune status of TNBC patients and risk scores, a single sample gene set enrichment analysis (SSGSEA) was implemented using "GSVA" package. The infiltration score of 16 immune cell subsets and 13 immune function pathways were compared between two risk groups. Immunophenoscore (IPS), a machine learning-based algorithm, was used for the quantitative evaluation of tumor immunogenicity. It was calculated according to the Z-score of representative cell type gene expression including: immunomodulators, effector cells, immunosuppressive cells and MHC molecules. The IPS ranges from 0 to 10 . A higher score indicates an increased immunogenicity and a better immunotherapy response. We retrieved patients' IPS from The Cancer Immunome Atlas (TCIA) database and compared them between two groups. At the same time, expression of four ICGs (PD1, CTLA-4, PD-L1, PD-L2) was also compared between two groups. 


\section{Establish a predictive nomogram combined with clinical parameters}

To evaluate whether this gene signature could serve as an independent prognostic predictor, univariate and multivariate Cox regression analysis were carried out. Variables were included in the model as covariates including: age, AJCC stage, whether the patients have taken surgery, radiotherapy or chemotherapy and risk score. Based on independent variables in both univariate and multivariate analysis, we established a nomogram by using "rms" R package. Next, calibration curves were further plotted to evaluate the consistency between the actual and predicted survival.

\section{Results}

\section{Differentially expressed immune-checkpoint genes in TNBC}

Among the 79 immune-checkpoint genes, we identified a total of 43 genes from TCGA dataset, and extracted the expression profile of these genes. Compared with normal tissues, 20 immune-checkpoint genes were differentially expressed in TNBC tumor tissues, among which 19 genes were upregulated and 1 gene was downregulated. The results were visualized using the heatmap (Figure $1 \mathrm{~A}$ ) and the volcano plot (Figure 1B).

\section{Functional enrichment analysis of DE-ICGs}

To further investigate functions and signaling pathways these DE-ICGs were involved in, GO and KEGG pathway analysis were conducted. The GO terms "T cell activation", "positive regulation of lymphocyte activation" and "regulation of leukocyte cell-cell adhesion" displayed significant enrichment in the category of biological process. For the category of cellular component, MHC protein complex was found to be enriched. For the molecular function, peptide antigen binding and antigen binding were the most abundant categories (Figure 2A). Meanwhile, KEGG pathway analysis revealed that these DE-ICGs were mainly associated with cell adhesion molecules, antigen processing and presentation, PD-L1 expression and PD-1 checkpoint pathway in cancer (Figure 2B).

\section{Identification of prognosis-related ICGs}

Firstly, univariate Cox regression analysis was used to identify candidate ICGs associated with overall survival (OS) of TNBC. 10 ICGs were identified as potential OS predictors of TNBC patients, including ID01, CD274, PDCD1LG2, PDCD1, CTLA4, ICOS, KIR3DL2, HLA-B, HLA-F, LGA3 (Figure 3A). All these genes were protective factors. Comparison of mRNA expression of these genes in normal and TNBC tissues was shown in Figure 3B, and correlation between ten genes was depicted in Figure 3C. Next, LASSO regression analysis showed that 3 of 10 ICGs were the OS-related ICGs including PDCD1LG2, KIR3DL2 and PDCD1 (Figure 3D-E). Finally, the 3 OS-related ICGs were included in the multivariant Cox regression model (Table 2). The coefficients of the 3 OS-related ICGs were shown in Figure 3F.

\section{Development and validation of an ICGs-based gene signature for individual survival prediction}


Based on coefficients and expression level of the 3 genes, the gene signature was generated: Risk

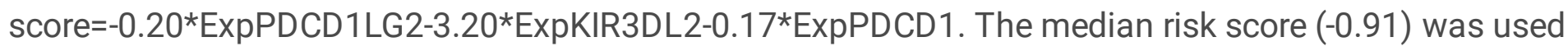
as cut-off point, and 113 patients were classified into two prognostic subgroups. As shown in Supplementary Figure 2, clinical parameters distribution and expression patterns of three genes were visualized in the form of heatmap between the two groups. The high-risk group tended to have advanced pathological stages and lower expression level of these hub genes. Next, survival analysis showed that patients in the high-risk group exhibited a worse prognosis ( $p=4.719 \mathrm{E}-04$, Figure $4 A)$. The distribution of risk score and survival status further corroborated the above conclusion (Figure 4B). We then evaluated the predictive accuracy of this gene signature by operating ROC curve analysis. The AUC for predicting 1-, 2- and 3-year OS was $0.925,0.822$ and 0.835 , respectively (Figure 4C). To validate the robustness of this model, we also applied this gene signature to the METABRIC cohort. In the validation cohort, the survival of low-risk patients was superior to high-risk patients (Figure 4D, E). Moreover, ROC curves also indicated a fairly good predictive value of 3-ICGs signature in the validation cohort (Figure 4F).

\section{Establish a nomogram for clinical application}

Univariate analysis showed that radiotherapy $(p=0.032)$, AJCC-stage $(p<0.001)$ and risk score $(p=0.003)$ were statistically significant in predicting the prognosis of TNBC patients (Figure 5A). Then, we investigated if the risk score was an independent predictor for clinical outcomes. As indicated by the multivariate analysis (Figure 5B), AJCC-stage $(p<0.001)$ and risk score $(p=0.018)$ were independent predictive factors for TNBC prognosis. By integrating above risk factors, we established a nomogram, which could facilitate clinicians to make risk quantification for TNBC patients (Figure 5C). By adding the points signed for each variable, we can obtain the total points and predict corresponding OS of each patient. Additionally, calibration curves revealed nomogram-predicted survival matched well with the actual observations, which laid a solid foundation for clinical application (Figure 5D-F).

\section{Patterns of immune cell infiltration and immunotherapy response of TNBC patients}

To evaluate the differences of immune status in two prognostic subgroups, enrichment scores of immune cell subsets and immune function pathways were quantified. As expected, adaptive immunity cells and innate immunity cells were evidently enriched in low-risk patients, demonstrating a high percentage of CD8 ${ }^{+} \mathrm{T}$ cells, B cells, DCs, macrophages and NK cells (Figure 6A). Higher enrichment scores were observed for cytokine-cytokine receptor interaction, check-point, cytolytic activity and T cell co-stimulation signaling pathways in low-risk group. Furthermore, the scores of type $\otimes$ and $\otimes$ IFN response were significantly higher than that in the high-risk group (Figure 6B). Altogether, these results indicated that effective immune response was more activated in the low-risk group, which may contribute to differences in the therapeutic efficacy. Meanwhile, we compared the immunogenicity in patients between two groups using IPS as a quantitative index for analysis. Our results showed that low-risk group obtained higher IPS of PD-1, CTLA4, PD-L1 and PD-L2 (Figure 6C). Similarly, the gene expression of CTLA4, PD-1, PD-L1 and PD-L2 was elevated in low-risk group (Figure 6D). We could speculate that low-risk group patients might get a favorable response to immunotherapy due to their higher immunogenicity. 


\section{Discussion}

In recent years, cancer immunotherapy has gained great attention. And its application in TNBC also shows new developments that hold the promise for improving outcomes of patients. Nonetheless, TNBC patients could hardly achieved complete pathological remission from therapy of ICls[12]. Therefore, it is critical to explore validated biomarkers to accurately screen out the population that might benefit from the immunotherapy. Considering the complexity of tumor heterogeneity, a single biomarker to predict prognosis and treatment response, appears not optimal and needs further improvement. The previous studies indicated that several immune checkpoint gene signature model had fairly good predictive value of long-term prognostic outcomes and therapeutic response in lung cancer[22], endometrial cancer[23] and hepatocellular carcinoma[20]. However, relevant studies in TNBC are not systematic and remain to be further explored. So, in this study, utilizing the TCGA and METABRIC database, we analyzed the expression of 43 immune checkpoint genes retrieved from literature, and screened out 3 genes independently associated with prognosis of TNBC patients. Based on above genes, a 3-gene signature was finally generated, which could be used to realize the individualized prediction of prognosis and immunotherapy response.

The novel gene signature was composed of three genes including PDCD1, PDCD1LG2 and KIR3DL2. Our findings suggested that all these genes were related to a favorable prognosis in patients with TNBC. PDCD1 (also known as PD-1) is an important immunosuppressive molecule, mostly expressed on activated $T$ cells and $B$ cells. It is responsible for mediating the immune escape of tumor cells and promoting tumor development, by binding to the ligands (PD-L1, PD-L2) located on the surface of tumor cells[24]. And antibodies targeting PD-1 have shown promising antitumor activity in melanoma[25], kidney cancer[26] and lung cancer[27]. Interestingly, however, some researchers noticed that PD-1 was also expressed on the surface of several tumor cell lines, which could contribute to anti-tumor effects by inhibiting AKT and ERK1/2 pathways[28]. In addition, it has also been suggested that PD-1 intrinsically expressed by tumor cells could be combined with self-expressed PD-L1 to better activate the immune system and trigger anti-tumor effects through this self-elimination approach[29]. Ren et al. conducted a study on 195 TNBC patients and reached the conclusion that patients with high PD-1 expression had favorable disease-free survival and overall survival[30], which was in agreement with our results. In previous studies, PD-L2 played different roles in different tumors. Xi et al. [31] showed that PD-L2 may be served as an indicator of poor prognosis in some digestive system tumors, while the opposite result was found in head and neck cancer[32]. Asano et al. [33] indicated that PD-L2 expression was not correlated with the prognosis of TNBC patients, which seemed not to be consistent with the findings of our study. However, the conclusion was limited by the small sample size. Similarly, KIR3DL2 could inhibit the tumorkilling ability by suppressing the secretion of IFN- $\gamma$ and TNF-a and the cytotoxic function of NK cells[34]. The effect of Lacutamab, an anti-KIR3DL2 monoclonal antibody, is being evaluated by the clinical trial with encouraging results[35, 36]. However, the role of KIR3DL2 in TNBC has not been reported and further research is required. 
With the risk signature, we could divide patients into low- and high-risk groups. Survival analysis revealed that the prognosis of low-risk group was clearly superior to the high-risk group, and ROC curve analysis reflected good predictive power of the gene signature. Meanwhile, we also testified the robustness of risk signature by external validation. In recent years, the nomogram-plot has been widely used in medical research and practice as a tool for prognosis evaluation. In our study, we developed a nomogram integrating three independent predictors for clinical outcomes, including risk signature and pathological stage. Consistently, the calibration curves showed the clinical value of nomogram was a reliable risk stratification tool.

The immune microenvironment is known to have a close relationship with the tumorigenicity and development of TNBC. Our findings showed that significantly abundant TILs, NK cells, CD8 ${ }^{+} \mathrm{T}$ cells, Tfh cells and B cells were observed in low-risk group patients determined by the 3-ICGs signature. The proportion of TILs in TNBC was closely related to the prognostic outcome[37]. Moreover, it was generally believed that enriched NK cells, B cells, $\mathrm{CD} 8^{+} \mathrm{T}$ cells were associated with favorable prognosis in TNBC patients. Likewise, Tfh cells played a key role in regulating the anti-tumor immune response of B cells[38]. In terms of immune signaling pathways, impaired immune activity was associated with a higher risk

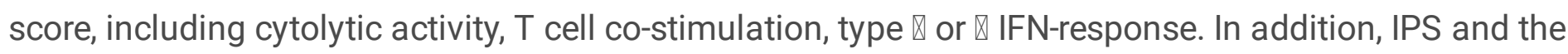
expression level of CTLA-4, PD-1, PD-L1 and PD-L2 were further investigated. IPS has been used to evaluate the response of tumors to immune checkpoint inhibitors[39]. For example, when atelizumab was approved for urothelial carcinoma and lung cancer, IPS was then defined as an evaluation index. Compared with patients in the high-risk group, our results showed that low-risk patients had higher expression of four representative ICGs and IPS. This suggested that low-risk patients were more likely to have higher immunogenicity and expected to benefit from immunotherapy. In clinical practice, for intractable breast cancer or recurrent or advanced breast cancer, the immune status of the patient's tumor microenvironment could be determined by detecting the expression levels of three genes in puncture specimens, which will be conducive to select an appropriate treatment plan, and develop a reasonable disease monitoring strategy.

In our study, we constructed an ICG-related prognostic gene signature for TNBC patients. The gene signature contained only three genes, and was more cost-effective and easier-to-use in clinical application. Meanwhile, external validation in the METABRIC database further confirmed the reliability of this novel gene signature. Moreover, from the immune status and IPS analysis, it could also be concluded that the gene signature may function as a potential immunotherapy response predictor. All these results will provide important guidance for clinical decision making. However, there were three limitations of our study. Firstly, this gene signature was constructed and validated based on public genomics databases, lacking prospective data to confirm these results. Secondly, the study subjects were dominated by North American population, and it was unclear whether this gene signature was equally valid for other ethnic population. Finally, the biological roles of genes in this gene signature remained to be further explored using experimental methods. Therefore, we would like to collect pathology specimens and clinical data 
from our own center for further validation and carry out more experimental work focusing on the function and biological mechanism of these hub genes in the future work.

\section{Declarations}

Acknowledgements: We acknowledge TCGA and METABRIC database for providing their platforms and contributions for uploading their meaningful datasets.

Authors' contributions: Conceived and designed the study: MS and NS; Data collection: YWL, XYZ and YL; Analyzed the data: JYL, SYT, and YWL; JYL, SYT, and YWL drafted the paper; all authors critically reviewed the manuscript.

Funding information: This study was funded by National Science Foundation for Young Scientists of China (No. 81801300).

Data availability: The data that support the findings of this study could be downloaded from TCGA (https://cancergenome.nih.gov/) and METABRIC database (http://molonc.bccrc.ca/apariciolab/research/metabric/).

\section{Compliance with ethical standards}

Research involving human participants and/or animals: Not applicable.

Conflicts of interest: The authors declare that they have no competing interests.

Informed Consent: For this type of study, formal consent is not required.

Ethical approval: This article does not contain any studies with human participants or animals performed by any of the authors.

Consent for publication: All authors consent to the publication of this study.

\section{References}

1. Siegel R, Miller K, Jemal A (2019) Cancer statistics, 2019. CA Cancer J Clin 69:7-34

2. Nadia H, Frédérique, Penault-Llorca J, vier (2019) Cortes, Michael, Gnant, Nehmat, Houssami, Breast cancer. Nature reviews. Disease primers 5:67-67

3. Bai X, Ni J, Beretov J, Graham P, Li Y (2021) Triple-negative breast cancer therapeutic resistance: Where is the Achilles' heel? Cancer Lett 497:100-111

4. Borri F, Granaglia A, Pathology of triple negative breast cancer. Semin. Cancer Biol. (2020)

5. Gluz O, Liedtke C, Gottschalk N, Pusztai L, Nitz U, Harbeck N (2009) Triple-negative breast cancercurrent status and future directions. Annals of oncology: official journal of the European Society for Medical Oncology 20:1913-1927 
6. Chang-Qing Y, Jie L, Shi-Qi Z, Kun Z, Zi-Qian G, Ran X, Hui-Meng L, Ren-Bin Z, Gang Z, Da-Chuan Y (2020) Z. Chen-Yan, Recent treatment progress of triple negative breast cancer. Prog Biophys Mol Biol 151:40-53

7. Schmid P, Adams S, Rugo H, Schneeweiss A, Barrios C, Iwata H, Diéras V, Hegg R, Im S, Shaw Wright G, Henschel V, Molinero L, Chui S, Funke R, Husain A, Winer E, Loi S (2018) L. Emens, Atezolizumab and Nab-Paclitaxel in Advanced Triple-Negative Breast Cancer. N Engl J Med 379:2108-2121

8. Garrido-Castro A, Lin N, Polyak K (2019) Insights into Molecular Classifications of Triple-Negative Breast Cancer: Improving Patient Selection for Treatment. Cancer Discov 9:176-198

9. Queirolo P, Boutros A, Tanda E, Spagnolo F, Quaglino P (2019) Immune-checkpoint inhibitors for the treatment of metastatic melanoma: a model of cancer immunotherapy. Semin Cancer Biol 59:290297

10. Ansell S, Lin Y (2020) Immunotherapy of lymphomas. J Clin Investig 130:1576-1585

11. Forde P, Chaft J, Smith K, Anagnostou V, Cottrell T, Hellmann M, Zahurak M, Yang S, Jones D, Broderick S, Battafarano R, Velez M, Rekhtman N, Olah Z, Naidoo J, Marrone K, Verde F, Guo H, Zhang J, Caushi J, Chan H, Sidhom J, Scharpf R, White J, Gabrielson E, Wang H, Rosner G, Rusch V, Wolchok J, Merghoub T, Taube J, Velculescu V, Topalian S, Brahmer J, Pardoll D, Neoadjuvant PD-1 Blockade in Resectable Lung Cancer. The New England journal of medicine 378 (2018) 1976-1986

12. Nanda R, Chow L, Dees E, Berger R, Gupta S, Geva R, Pusztai L, Pathiraja K, Aktan G, Cheng J, Karantza V, Buisseret L (2016) Pembrolizumab in Patients With Advanced Triple-Negative Breast Cancer: Phase lb KEYNOTE-012 Study. Journal of clinical oncology: official journal of the American Society of Clinical Oncology 34:2460-2467

13. Heimes A, Schmidt M (2019) Atezolizumab for the treatment of triple-negative breast cancer. Expert opinion on investigational drugs 28:1-5

14. Emens L, Ascierto P, Darcy P, Demaria S, Eggermont A, Redmond W, Seliger B, Marincola F, Cancer immunotherapy: Opportunities and challenges in the rapidly evolving clinical landscape. European journal of cancer (Oxford, England (1990) 81 (2017) 116-129

15. Santarpia M, González-Cao M, Viteri S, Karachaliou N, Altavilla G, Rosell R (2015) Programmed cell death protein-1/programmed cell death ligand-1 pathway inhibition and predictive biomarkers: understanding transforming growth factor-beta role. Translational lung cancer research 4:728-742

16. Luen S, Virassamy B, Savas P, Salgado R, Loi S (2016) The genomic landscape of breast cancer and its interaction with host immunity. Breast 29:241-250

17. Schumacher T, Schreiber R (2015) Neoantigens in cancer immunotherapy. Science 348:69-74

18. Keenan T, Tolaney S (2020) Role of Immunotherapy in Triple-Negative Breast Cancer. Journal of the National Comprehensive Cancer Network: JNCCN 18:479-489

19. Song D, Tian J, Han X, Li X (2021) A model of seven immune checkpoint-related genes predicting overall survival for head and neck squamous cell carcinoma. European archives of oto-rhinolaryngology: official journal of the European Federation of Oto-Rhino-Laryngological Societies (EUFOS) : affiliated with the German Society for Oto-. Rhino-Laryngology - Head and Neck Surgery 
20. Xu D, Liu X, Wang Y, Zhou K, Wu J, Chen J, Chen C, Chen L, Zheng J (2020) Identification of immune subtypes and prognosis of hepatocellular carcinoma based on immune checkpoint gene expression profile, 126. Biomedicine \& pharmacotherapy = Biomedecine \& pharmacotherapie, p 109903

21. Hu F, Liu C, Liu L, Zhang Q, Guo A, Expression profile of immune checkpoint genes and their roles in predicting immunotherapy response. Brief. Bioinform. (2020)

22. Yi M, Li A, Zhou L, Chu Q, Luo S, Wu K (2021) Immune signature-based risk stratification and prediction of immune checkpoint inhibitor's efficacy for lung adenocarcinoma. Cll, Cancer immunology

23. Liu J, Nie S, Wu Z, Jiang Y, Wan Y, Li S, Meng H, Zhou S, Cheng W (2020) Exploration of a novel prognostic risk signatures and immune checkpoint molecules in endometrial carcinoma microenvironment. Genomics 112:3117-3134

24. Pardoll M, Drew (2012) The blockade of immune checkpoints in cancer immunotherapy. Nat Rev Cancer 12:252-264

25. Menzies A, Amaria R, Rozeman E, Huang A, Tetzlaff M, van de Wiel B, Lo S, Tarhini A, Burton E, Pennington T, Saw R, Xu X, Karakousis G, Ascierto P, Spillane A, van Akkooi A, Davies M, Mitchell T, Tawbi H, Scolyer R, Wargo J, Blank C, Long G (2021) Pathological response and survival with neoadjuvant therapy in melanoma: a pooled analysis from the International Neoadjuvant Melanoma Consortium (INMC). Nat Med 27:301-309

26. Flippot R, Escudier B, Albiges L (2018) Immune Checkpoint Inhibitors: Toward New Paradigms in Renal Cell Carcinoma. Drugs 78:1443-1457

27. Mazieres J, Drilon A, Lusque A, Mhanna L, Cortot A, Mezquita L, Thai A, Mascaux C, Couraud S, Veillon R, Van den Heuvel M, Neal J, Peled N, Früh M, Ng T, Gounant V, Popat S, Diebold J, Sabari J, Zhu V, Rothschild S, Bironzo P, Martinez-Marti A, Curioni-Fontecedro A, Rosell R, Lattuca-Truc M, Wiesweg M, Besse B, Solomon B, Barlesi F, Schouten R, Wakelee H, Camidge D, Zalcman G, Novello S, Ou S, Milia J, Gautschi O (2019) Immune checkpoint inhibitors for patients with advanced lung cancer and oncogenic driver alterations: results from the IMMUNOTARGET registry. Annals of oncology: official journal of the European Society for Medical Oncology 30:1321-1328

28. Wang $X$, Yang X, Zhang C, Wang Y, Cheng T, Duan L, Tong Z, Tan S, Zhang H, Saw P, Gu Y, Wang J, Zhang Y, Shang L, Liu Y, Jiang S, Yan B, Li R, Yang Y, Yu J, Chen Y, Gao G, Ye Q, Gao S, Tumor cellintrinsic PD-1 receptor is a tumor suppressor and mediates resistance to PD-1 blockade therapy. Proc. Natl. Acad. Sci. U. S. A. 117 (2020) 6640-6650

29. Zhao Y, Harrison DL, Song Y, Ji J, Huang J (2018) Antigen-Presenting Cell-Intrinsic PD-1 Neutralizes PD-L1 in cis to Attenuate PD-1 Signaling in T Cells. Cell Reports 24:379

30. Ren X, Wu H, Lu J, Zhang Y, Luo Y, Xu Q, Shen S, Liang Z (2018) PD1 protein expression in tumor infiltrated lymphocytes rather than PDL1 in tumor cells predicts survival in triple-negative breast cancer. Cancer Biol Ther 19:373-380

31. XI A, KI B, Cl C, Jw B, Yt B, Prognostic and clinicopathological utility of PD-L2 expression in patients with digestive system cancers: A meta-analysis. Int. Immunopharmacol. 88 
32. Yearley JH, Gibson C, Yu N, Moon C, Murphy E, Juco J, Lunceford J, Cheng J, Chow L, Seiwert TY (2017) PD-L2 Expression in Human Tumors: Relevance to Anti-PD-1 Therapy in Cancer. Clin Cancer Res 51:3158-3167

33. Asano Y, Kashiwagi S, Goto W, Takada K, Takahashi K, Morisaki T, Fujita H, Takashima T, Tomita S, Ohsawa M (2018) Prediction of treatment responses to neoadjuvant chemotherapy in triple-negative breast cancer by analysis of immune checkpoint protein expression. J Transl Med 16:87

34. Martinez X, Di Raimondo C, Abdulla F, Zain J, Rosen S, Querfeld C (2019) Leukaemic variants of cutaneous T-cell lymphoma: Erythrodermic mycosis fungoides and Sézary syndrome. Best practice \& research. Clinical haematology 32:239-252

35. Bagot M, Porcu P, Marie-Cardine A, Battistella M, William B, Vermeer M, Whittaker S, Rotolo F, RamWolff C, Khodadoust M, Bensussan A, Paturel C, Bonnafous C, Sicard H, Azim H, Kim Y (2019) IPH4102, a first-in-class anti-KIR3DL2 monoclonal antibody, in patients with relapsed or refractory cutaneous T-cell lymphoma: an international, first-in-human, open-label, phase 1 trial. Lancet Oncol 20:1160-1170

36. Khan M, Arooj S, Wang H, NK Cell-Based Immune Checkpoint Inhibition. Front. Immunol. 11 (2020)

37. Loi S, Michiels S, Salgado R, Sirtaine N, Jose V, Fumagalli D, Kellokumpu-Lehtinen P, Bono P, Kataja V, Desmedt C, Piccart M, Loibl S, Denkert C, Smyth M, Joensuu H, Sotiriou C (2014) Tumor infiltrating lymphocytes are prognostic in triple negative breast cancer and predictive for trastuzumab benefit in early breast cancer: results from the FinHER trial. Annals of oncology: official journal of the European Society for Medical Oncology 25:1544-1550

38. Hollern D, Xu N, Thennavan A, Glodowski C, Garcia-Recio S, Mott K, He X, Garay J, Carey-Ewend K, Marron D, Ford J, Liu S, Vick S, Martin M, Parker J, Vincent B, Serody J, Perou C (2019) B Cells and T Follicular Helper Cells Mediate Response to Checkpoint Inhibitors in High Mutation Burden Mouse Models of Breast Cancer. Cell 179:1191-1206.e1121

39. Charoentong P, Finotello F, Angelova M, Mayer C, Efremova M, Rieder D, Hackl H, Trajanoski Z (2017) Pan-cancer Immunogenomic Analyses Reveal Genotype-Immunophenotype Relationships and Predictors of Response to Checkpoint Blockade. Cell Rep 18:248-262

\section{Tables}

Table 1. Baseline characteristics of TNBC patients in the TCGA and METABRIC cohort. 


\begin{tabular}{|c|c|c|}
\hline Characteristics & TCGA group $\mathrm{N}=113[\%]$ & METABRIC group $\mathrm{N}=286 \square \% \square$ \\
\hline \multicolumn{3}{|c|}{ Age at diagnosis (years) } \\
\hline$\otimes 50$ & $41(36.3 \%)$ & $104(36.4 \%)$ \\
\hline$\geq 50$ & $72(63.7 \%)$ & $182(63.6 \%)$ \\
\hline \multicolumn{3}{|l|}{ T-stage } \\
\hline T1-2 & $101(89.4 \%)$ & $264(92.3 \%)$ \\
\hline T3-4 & $12(10.6 \%)$ & $22(7.7 \%)$ \\
\hline \multicolumn{3}{|l|}{$\mathrm{N}$-stage } \\
\hline NO & $72(63.7 \%)$ & NA \\
\hline $\mathrm{N}+$ & $41(36.3 \%)$ & NA \\
\hline \multicolumn{3}{|l|}{ AJCC stage } \\
\hline I & $18(15.9 \%)$ & $104(36.4 \%)$ \\
\hline II & $74(65.5 \%)$ & $58(20.3 \%)$ \\
\hline III & $19(16.8 \%)$ & $43(15.0 \%)$ \\
\hline IV & $2(1.8 \%)$ & $0(0.0 \%)$ \\
\hline unknown & $0(0.0 \%)$ & $81(28.3 \%)$ \\
\hline \multicolumn{3}{|l|}{ Surgery } \\
\hline No & $5(4.4 \%)$ & $6(2.1 \%)$ \\
\hline Yes & $108(95.6 \%)$ & $280(97.9 \%)$ \\
\hline \multicolumn{3}{|l|}{ Radiotherapy } \\
\hline No & $51(45.1 \%)$ & $80(28.0 \%)$ \\
\hline Yes & $62(54.9 \%)$ & $206(72.0 \%)$ \\
\hline \multicolumn{3}{|l|}{ chemotherapy } \\
\hline No & $34(30.1 \%)$ & $132(46.2 \%)$ \\
\hline Yes & 79 (69.9\%) & 154 (53.8\%) \\
\hline
\end{tabular}

Table 2. Description and risk coefficient of hub genes. 


\begin{tabular}{|llc|}
\hline $\begin{array}{l}\text { Gene } \\
\text { symbol }\end{array}$ & Description & $\begin{array}{c}\text { Risk } \\
\text { coefficient }\end{array}$ \\
\hline PDCD1LG2 & Programmed Cell Death 1 Ligand 2 & -0.199675855 \\
\hline KIR3DL2 & $\begin{array}{l}\text { Killer Cell Immunoglobulin Like Receptor, Three Ig Domains And Long } \\
\text { Cytoplasmic Tail 2 }\end{array}$ & -3.200022817 \\
\hline & Programmed Cell Death 1 & \\
\hline PDCD1 & & -0.171493072 \\
\hline
\end{tabular}

\section{Figures}

A

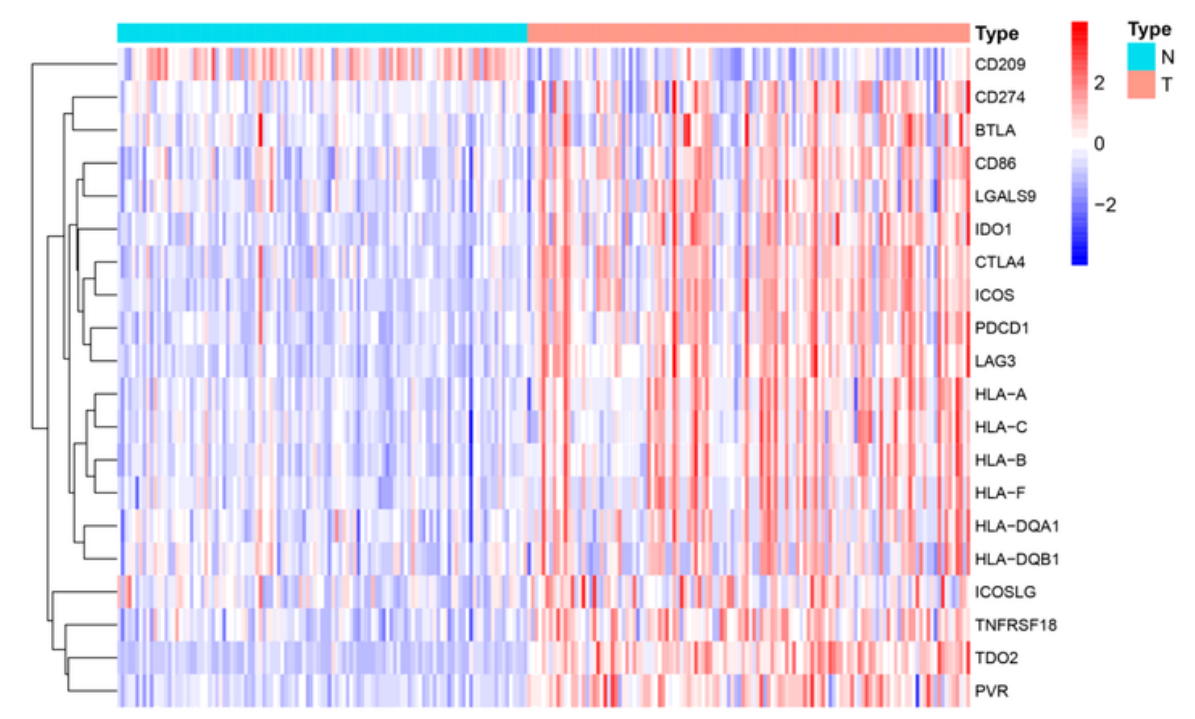

B

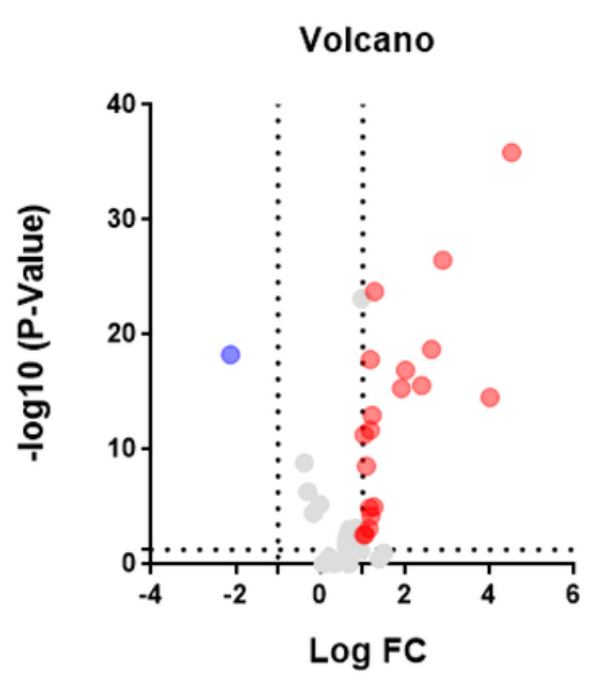

Figure 1

Identification of DE-ICGs in TNBC tissues from TCGA database. (A) Heatmap of DE-ICGs. (B) Volcano plot of DE-ICGs; red dot represents up-regulated gene, and blue dot represent down-regulated gene. 


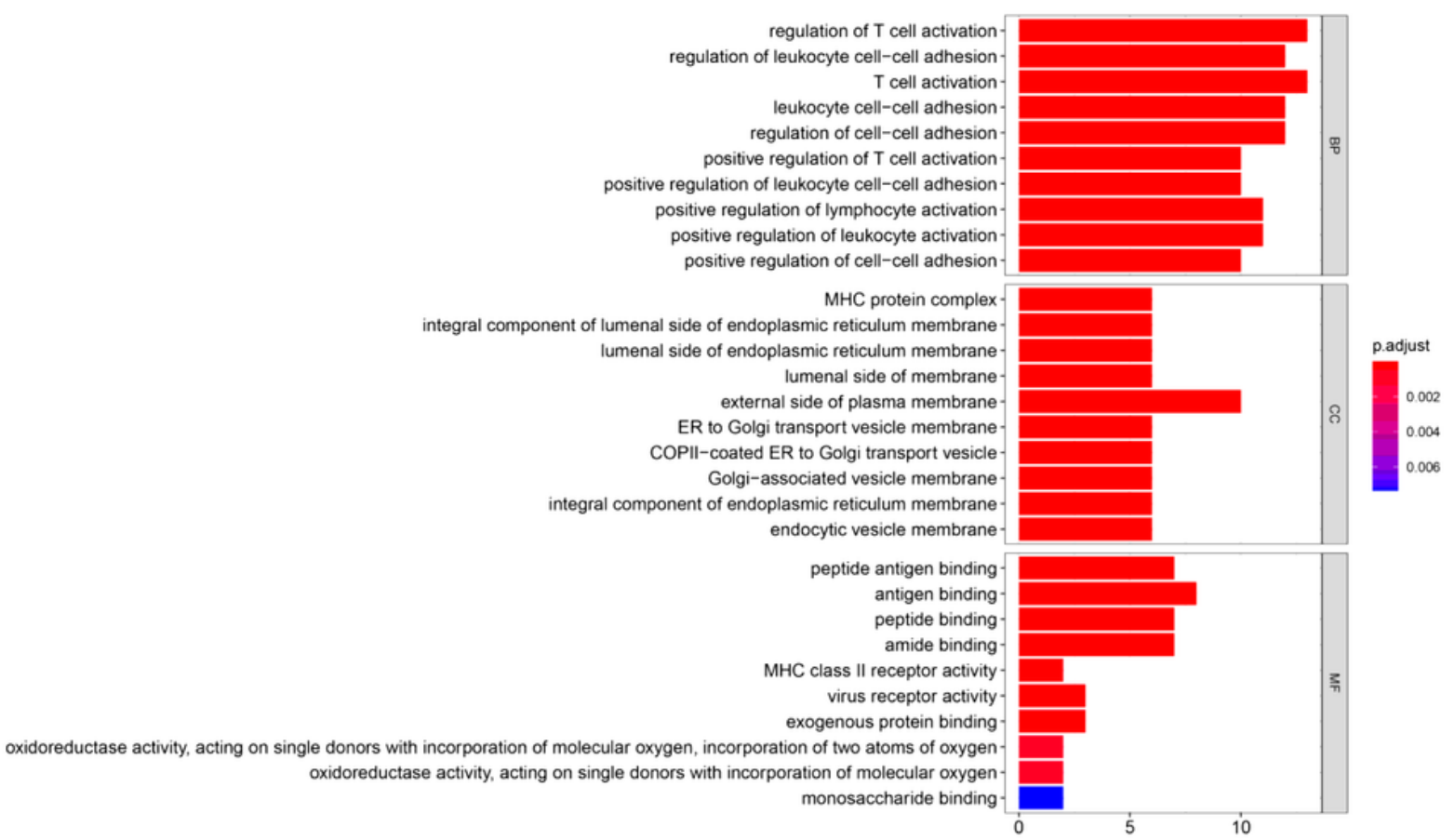

B

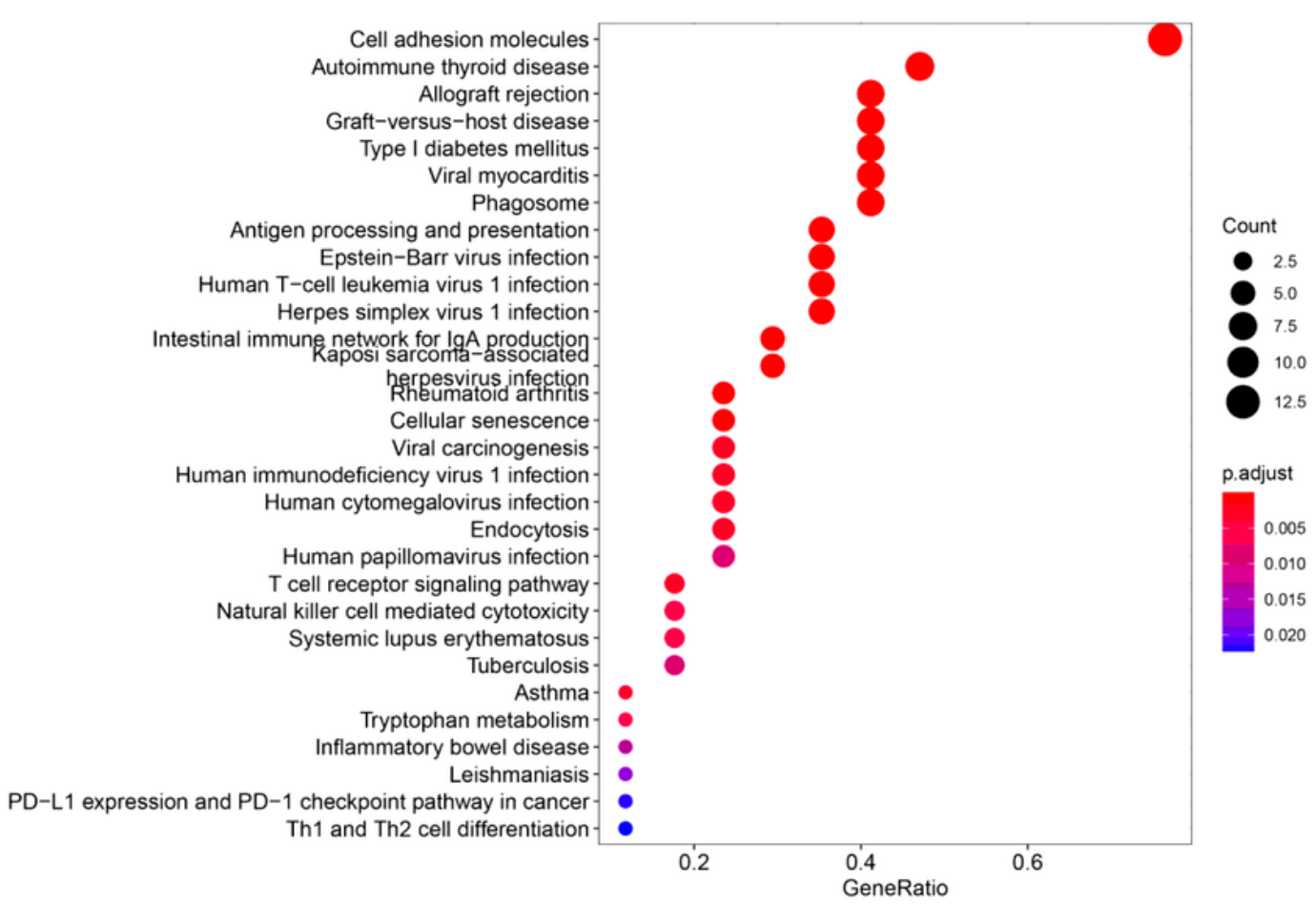

Figure 2

Functional enrichment analysis of DE-ICGs. (A) GO enrichment analysis of DE-ICGs. (B) KEGG pathway enrichment analysis of DE-ICGs. 
A

B

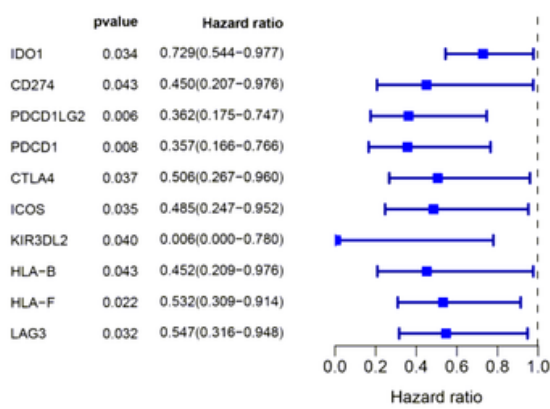

D

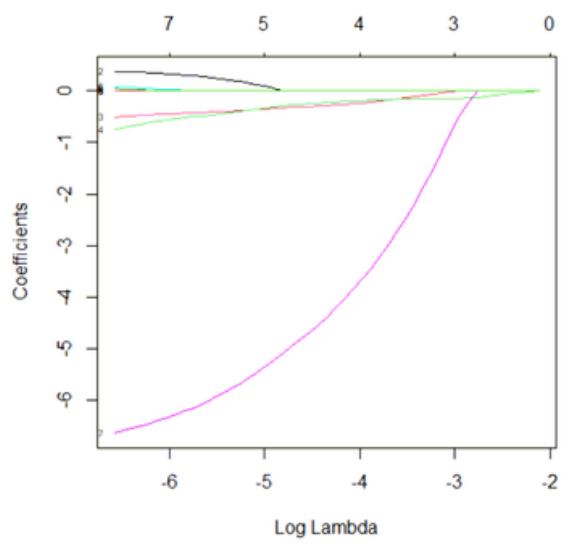

type 白 $\mathrm{N}$ 白 $\mathrm{T}$

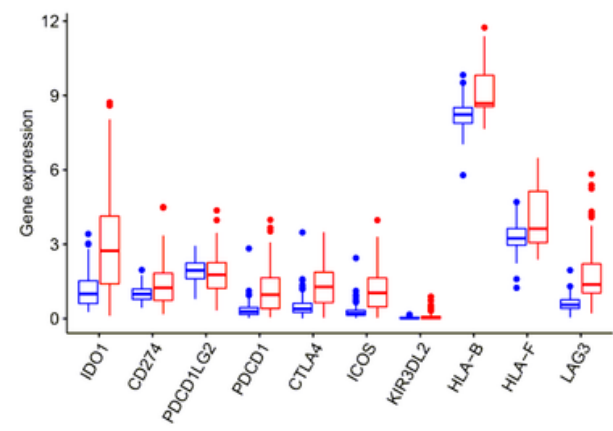

$\mathbf{E}$

$\begin{array}{lllllllllllllllll}9 & 8 & 7 & 6 & 5 & 5 & 5 & 5 & 4 & 4 & 3 & 3 & 3 & 2 & 1 & 1 & 0\end{array}$

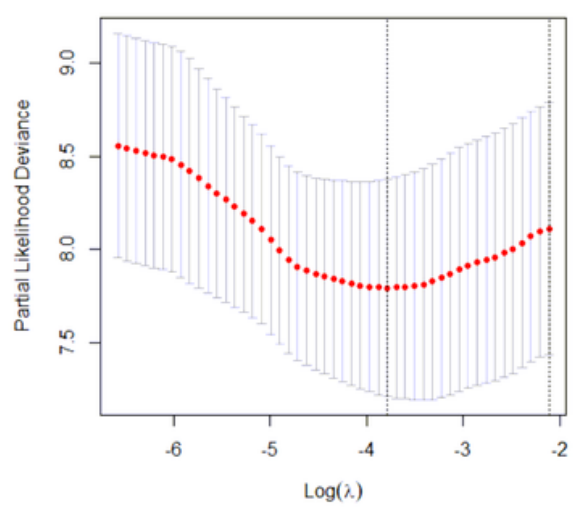

C

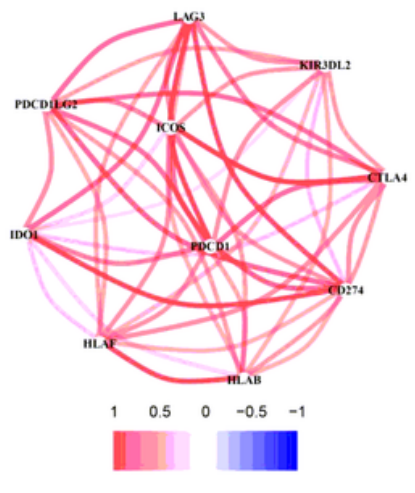

$\mathbf{F}$

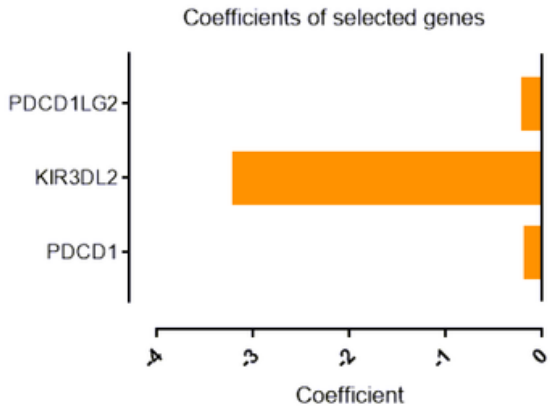

Figure 3

Identification of prognosis-related ICGs. (A) Forest plot demonstrating univariate Cox regression analysis of ICGs related to OS. (B) Boxplot of ICGs associated with OS. (C) The correlation network of ten OSrelated ICGs. (D-E) LASSO-Cox regression analysis to select independent prognostic genes. (F) Histogram showing the risk coefficients of three hub genes. 
A

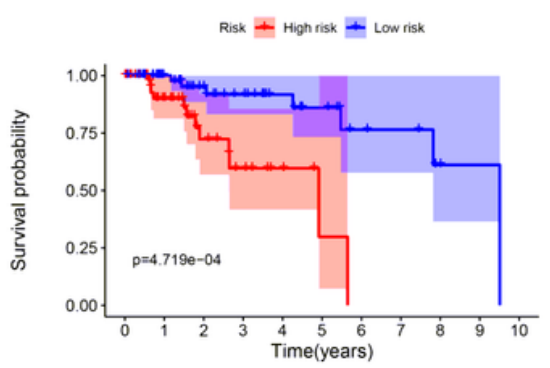

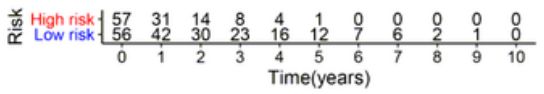

D
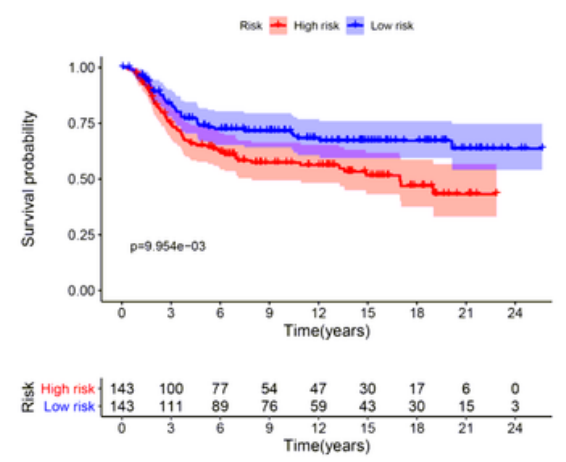

B

Training cohort
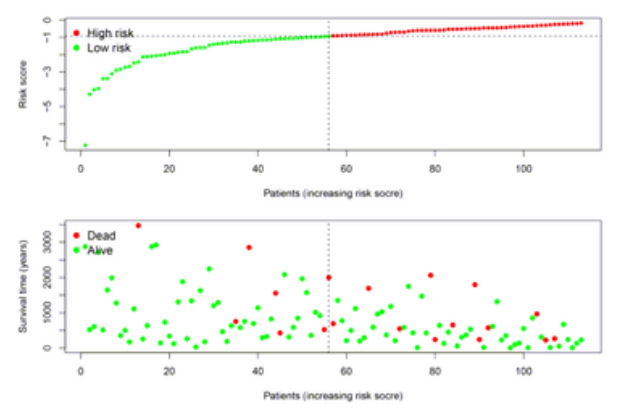

Validation cohort

E
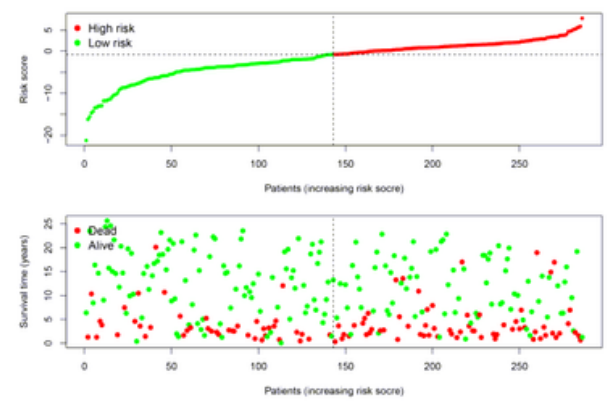

Training cohort

C

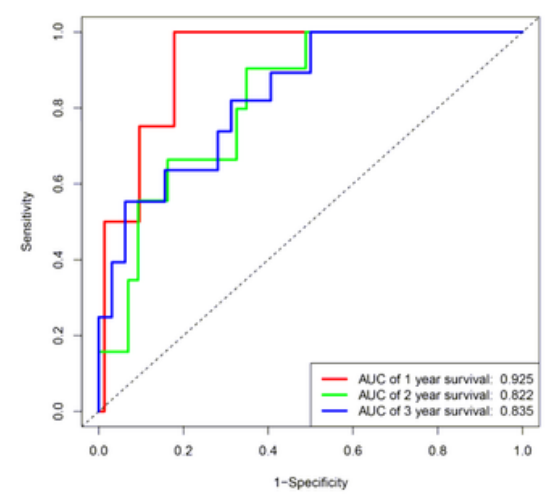

Validation cohort

$\mathbf{F}$

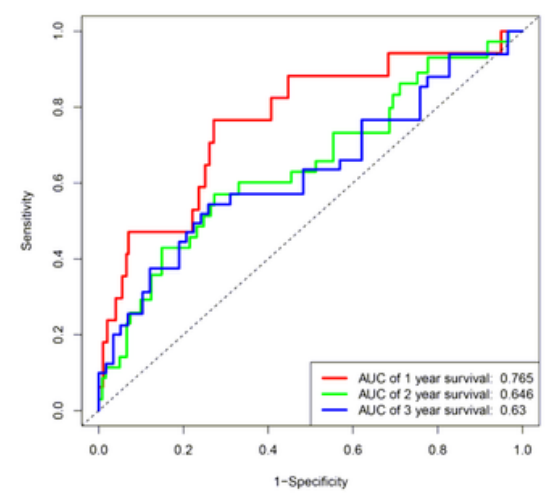

\section{Figure 4}

Development and validation of the prognostic gene signature based on ICGs. (A) Survival analysis of two groups by Kaplan-Meier curves in TCGA cohort. (B) Distribution of survival status and risk score in the TCGA cohort. (C) 1-year, 2-year, and 3-year ROC curves of the gene signature in the TCGA cohort. (D) Survival analysis of two groups by Kaplan-Meier curves in external METABRIC cohort. (E) Distribution of survival status and risk score in the METABRIC cohort. (F) 1-year, 2-year, and 3-year ROC curves of the gene signature in the METABRIC cohort. 
A

$\begin{array}{lrr} & \text { p value } & \text { Hazard ratio } \\ \text { age } & 0.791 & 1.134(0.446-2.885) \\ \text { surgery } & 0.204 & 0.432(0.119-1.576) \\ \text { radiotherapy } & 0.032 & 0.350(0.134-0.915) \\ \text { chemotherapy } & 0.066 & 0.390(0.143-1.065) \\ \text { A.JCC-stage } & <0.001 & 4.833(2.290-10.200) \\ \text { Risk score } & 0.003 & 8.796(2.123-36.446)\end{array}$

B

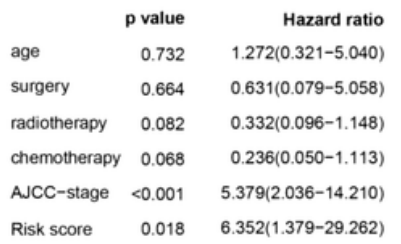

Risk score $\quad 0.018 \quad 6.352(1.379-29.262)$
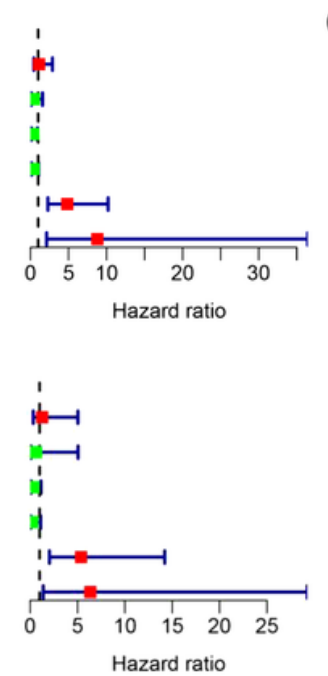

C

Points

AJCC-stage

Risk score

Total Points

1-year survival

3-year survival

5-year survival
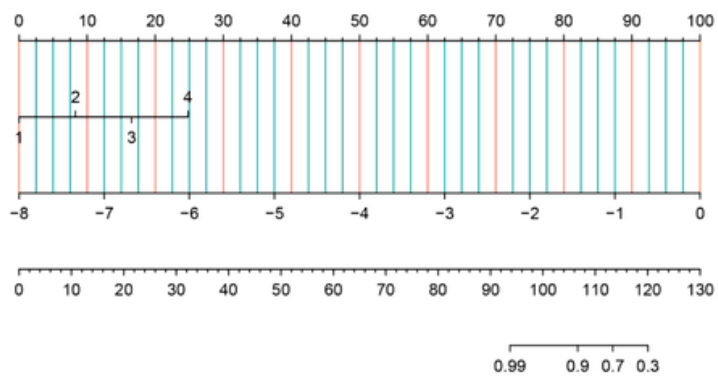

$\begin{array}{llllll}0.99 & 0.9 & 0.7 & 0.3 & 0.01\end{array}$

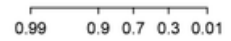

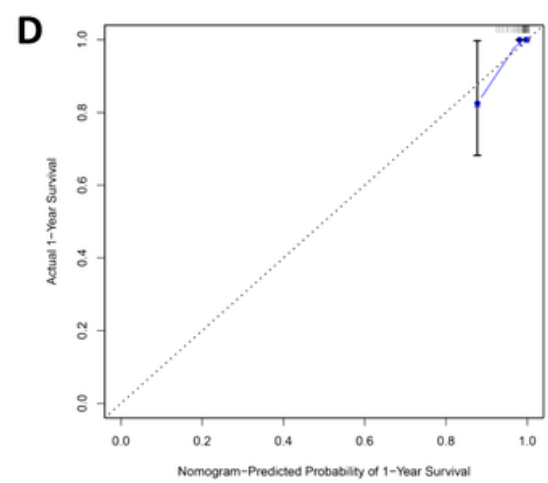
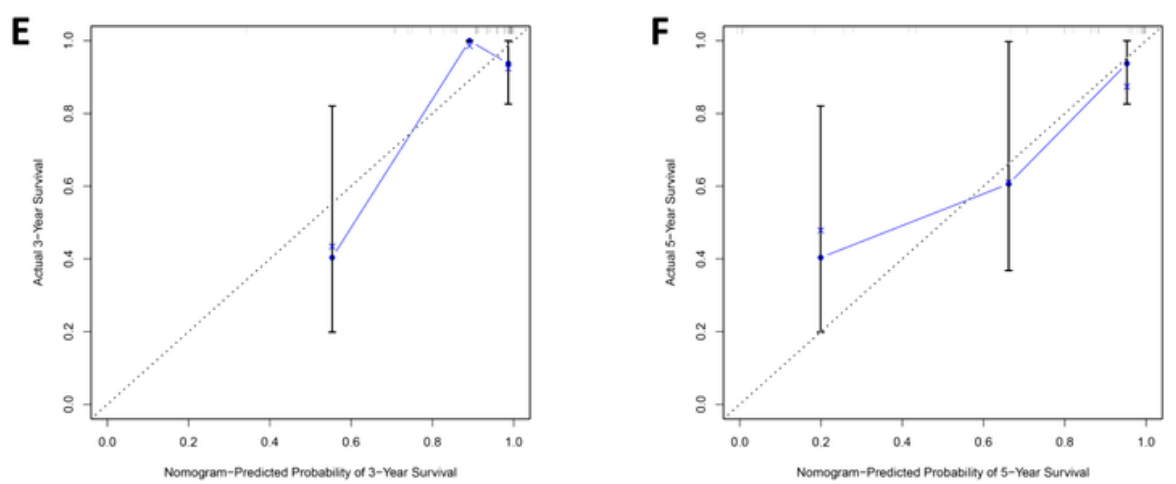

\section{Figure 5}

Establishing a prognostic nomogram foe TNBC patients. (A-B) Univariate and multivariate analyses for survival of TNBC. (C) The nomogram for predicting the OS of TNBC. (D-F) Calibration curves for predicting OS of TNBC at 1-year, 2-year, and 3-year. 
A

Risk 官 low 官 high

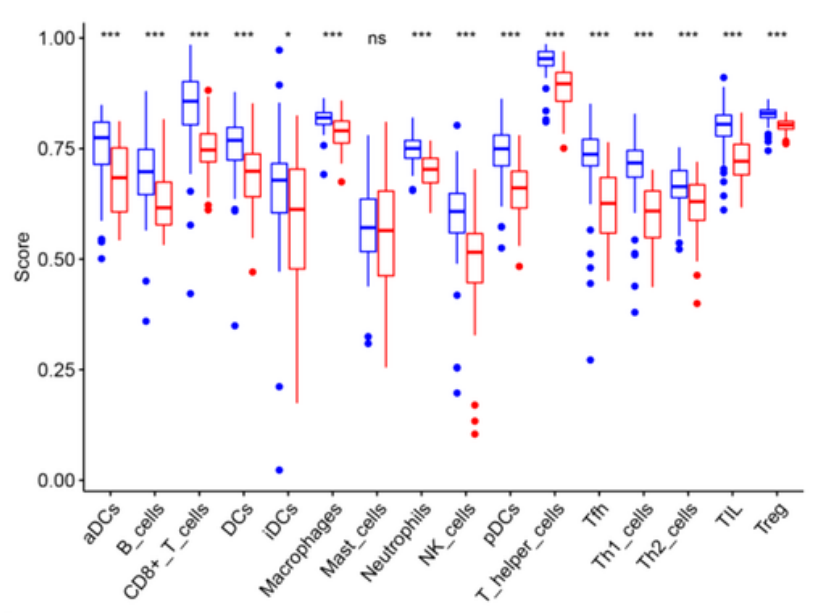

C

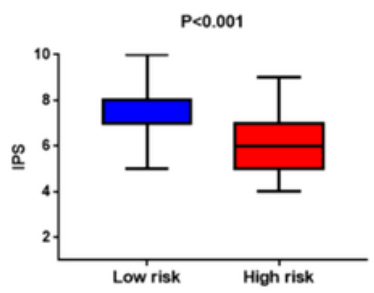

D

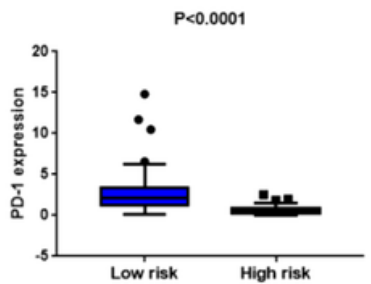

B
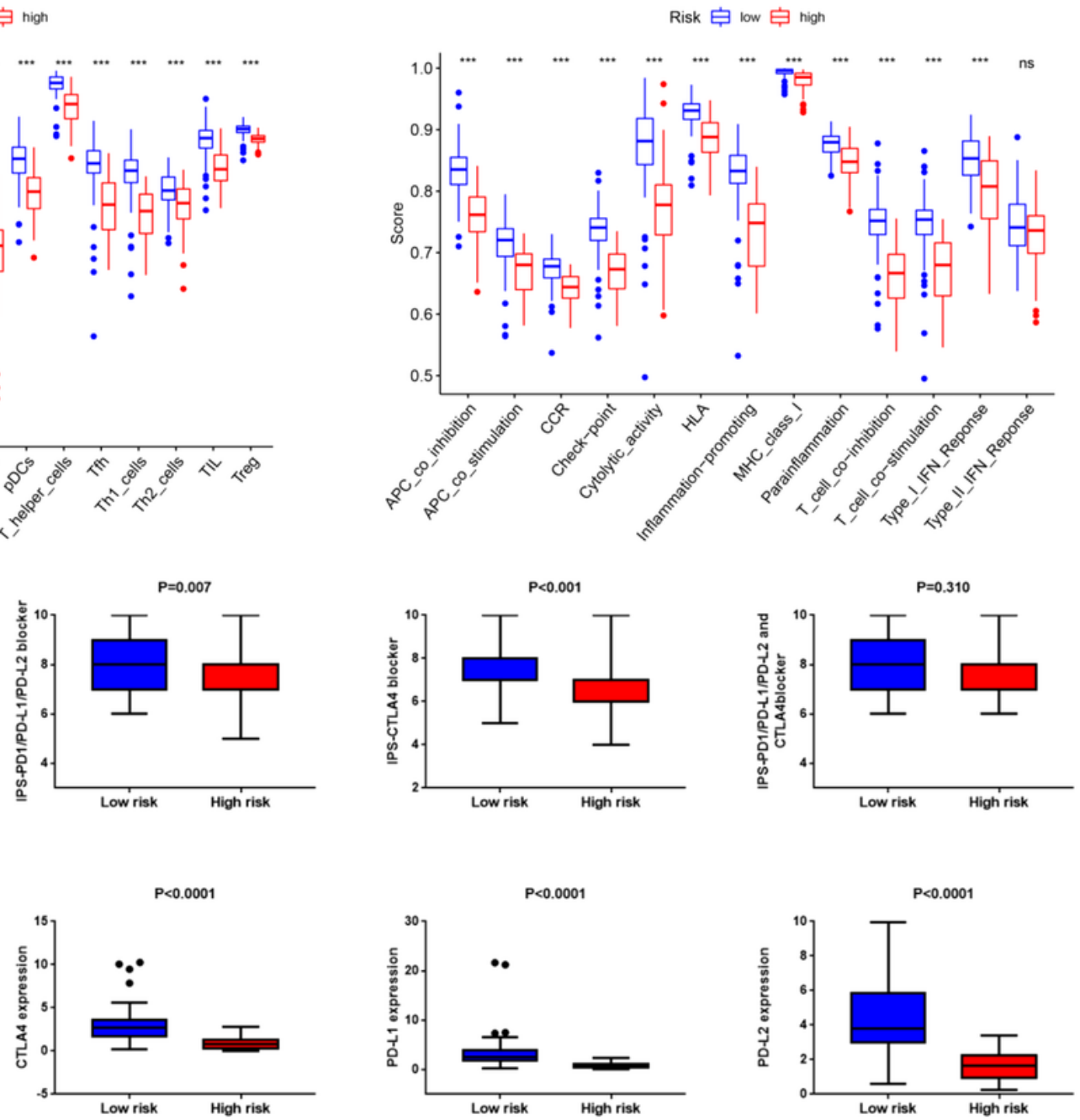

\section{Figure 6}

Association between gene signature and infiltration of immune cells and immunotherapy response. (A-B) Comparison of immune infiltrating cells and immune-related pathways between patients of low risk and high risk. (C) Correlation analysis of risk signature and IPS. (D) Expression of four immune checkpoint genes in two groups.

\section{Supplementary Files}

This is a list of supplementary files associated with this preprint. Click to download.

- SupplementaryFigure1.tif

- SupplementaryFigure2.tif

- SupplementaryTable1.docx 